\title{
Location or origin? What is critical for macrophage propagation of lung fibrosis?
}

\author{
Xiaofeng Zhou ${ }^{1}{ }^{1}$ and Bethany B. Moore ${ }^{1,2}$ \\ Affiliations: ${ }^{1}$ Dept of Internal Medicine, Pulmonary and Critical Care Medicine Division, University of Michigan, \\ Ann Arbor, MI, USA. ${ }^{2}$ Dept of Microbiology and Immunology, University of Michigan, Ann Arbor, MI, USA.
}

Correspondence: Xiaofeng Zhou, 4062 BSRB, 109 Zina Pitcher Place, Ann Arbor, MI 48109-2200, USA.

E-mail: xiazhouQumich.edu

@ERSpublications

Recruited lung macrophages are key in driving lung fibrosis, but the location varies with different forms of fibrosis http://ow.ly/kTIq30ixgpR

Cite this article as: Zhou X, Moore BB. Location or origin? What is critical for macrophage propagation of lung fibrosis? Eur Respir J 2018; 51: 1800103 [https://doi.org/10.1183/13993003.00103-2018].

Radiation-induced pulmonary fibrosis develops following radiotherapy for chest wall and lung malignancies, affects quality of life and is often lethal [1]. Similarly, idiopathic forms of lung fibrosis show progressive accumulation of extracellular matrix leading to respiratory insufficiency [2]. Currently, treatments for either radiation-induced or idiopathic forms of pulmonary fibrosis are limited and largely ineffective. Immunosuppressive therapies may be harmful in idiopathic pulmonary fibrosis [3], and are used in radiation-induced fibrosis with limited effect [4]. Growing evidence suggests macrophages are critical regulators of lung fibrosis [5]; however, the subtypes involved and mechanisms responsible are just beginning to be understood. Pulmonary macrophages are critical for innate immunity and response to pathogens or injury, but also play important roles in resolution of inflammation and wound healing. These versatile immune cells are composed of heterogeneous populations with different origins that reside in unique locations within the lung.

Pulmonary tissue macrophages can be divided into subsets based on anatomic locations: alveolar macrophages (AMs) and interstitial macrophages (IMs). AMs and IMs are separated based on differential expression of CD11c and CD11b [6,7] and colony stimulating factor receptor (CSFR) subtypes [8]. Residential AMs originate from the yolk sac during embryogenesis and are long-lived cells that self-renew during homeostasis $[9,10]$. Additionally, AMs originate postnatally from circulating monocytes recruited via a CCL2/CCR2 axis $[11,12]$. A study comparing resident and recruited AMs during experimental acute lung injury found recruited AMs enriched for immune signalling, inflammation and glycolytic and arginine metabolism, whereas resident AMs were characterised by proliferation, tricarboxylic acid cycle, amino acids and fatty acid metabolism pathways [12].

IMs are derived from both yolk sac macrophages and bone marrow-derived monocytes [13], and can be replenished by circulating monocytes $[8,14]$. Three distinct populations of IMs are distinguished by surface markers [8] and turnover rates. Collectively, IM populations comprise $\sim 9 \%$ of extravascular myeloid cells in the lung, whereas AMs constitute $\sim 75 \%$ of this pool of cells [8]. Notably, expression of CSF2R is prominent on AMs while CSF1R characterises all IM subtypes which are long-lived during homeostasis but do eventually replenish from circulation [8].

Received: Jan 162018 | Accepted after revision: Feb 202018

Support statement: This work was supported by the NIH Center for Scientific Review (grant no. R01 HL127805). Funding information for this article has been deposited with the Crossref Funder Registry.

Conflict of interest: X. Zhou received grants from NIH, during the conduct of the study. B.B. Moore received grants from NIH, during the conduct of the study.

Copyright OERS 2018 
Macrophages are often found in close proximity to fibrogenic sites and may contribute to chronic inflammation to promote progressive fibrosis. Recent studies pinpoint roles of specific macrophage populations in different forms of fibrogenesis, but use of different markers to classify AMs versus IMs and different depletion methods in various studies make comparisons challenging. In addition, upon injury, monocytes are recruited into the lung, and differentiate into monocyte-derived macrophages that can enter both alveolar or interstitial spaces [11]. In bleomycin-induced pulmonary fibrosis, monocyte-derived (recruited) AMs, rather than tissue-resident AMs or IMs, are required for development of fibrosis and the transcriptome of recruited versus resident AMs differ significantly [11]. Conversely, in a repeated injury of type II alveolar epithelial cell-induced fibrosis model, Ly6C $\mathrm{C}^{\mathrm{hi}}$ monocyte-derived non-resident macrophages drive fibrosis [15]. In this issue of the European Respiratory Journal, Meziani et al. [16] identified Gr-1 ${ }^{-}$ IMs as the pulmonary macrophage population necessary for development of radiation-induced fibrosis. If you are counting, that is three different forms of lung fibrosis implicating three different types of lung macrophages found in both alveolar and interstitial spaces as drivers of disease. So, is it location, origin or activation phenotype of macrophages that matter?

M1/M2 polarisation was originally defined by differential responses of macrophages to in vitro stimulation with interferon- $\gamma$ or interleukin-4 $[17,18]$. M1 macrophages mediate resistance to pathogens, but also contribute to tissue destruction, while M2 macrophages are less toxic to microbes and host cells, have anti-inflammatory and reparative functions [19], but are often implicated in aberrant wound-healing leading to fibrosis in kidneys, bladder, liver and lungs [20]. However, this M1/M2 paradigm represents extremes of the spectrum, while macrophages activated in vivo may obtain mixed phenotypes $[11,12,21]$. In addition, pulmonary macrophages are highly plastic and may obtain transient activation phenotypes instead of achieving terminally differentiated states [5].

MeZiani et al. [16] found that both AMs and IMs increased at 20 weeks post-radiation when fibrosis is progressing. The IMs display a strong M2 phenotype at 20 weeks as evidenced by up-regulation of CD206, a 400-fold increase in arginase mRNA and down-regulation of M1 markers. In contrast, AMs show mixed M1/M2 phenotypes. Taking advantage of the ability of clodronate given intranasally to deplete both resident and recruited AMs versus ability of CSF1R neutralisation to specifically deplete IMs, this study convincingly showed depletion of IMs from week 15-20 post-radiation exposure limits development of radiation-induced lung fibrosis. The authors also found increased infiltration of macrophages with M2-like phenotypes in parenchyma of human radiation-induced pulmonary fibrosis. Strikingly, in a co-culture system only IMs isolated from fibrotic lungs, but not AMs, could induce fibroblasts to produce $\alpha$-smooth muscle actin and transforming growth factor- $\beta$, hallmarks of myofibroblast differentiation. Taken together these data establish the critical role of IMs in this model.

Involvement of distinct macrophage populations in different pulmonary fibrosis models may reflect different pathways of fibrogenesis. Radiation-induced pulmonary fibrosis develops over 20 weeks and involves damage to lung parenchyma as well as alveolar spaces, while intratracheal bleomycin or repetitive injury of type II alveolar epithelium leads to fibrosis in 2-3 weeks with damage likely limited to lung epithelium. Furthermore, bleomycin-induced pulmonary fibrosis is self-limiting after 28 days and often resolves in 8-12 weeks [22]. Interestingly, following bleomycin treatment monocytes continuously differentiate to recruited AMs, but following resolution these monocyte-derived AMs become increasingly similar to tissue-resident AMs in gene expression profiles [11]. Similarly, IMs increase during radiation-induced pulmonary fibrosis [16], suggesting accumulation of monocyte-derived IMs. Thus, it appears the common theme may be macrophages associated with lung fibrosis pathology most often derive from the circulating monocytes regardless of location in alveolar versus interstitial space. We believe the location and duration of the injury are factors which influence where these cells accumulate eventually in the different forms of lung fibrosis.

If origin dictates pathogenesis, this raises the possibility of directed therapeutics by blocking recruitment of monocyte-derived macrophages. CCR2 depletion was first shown to limit bleomycin-induced fibrosis in 2001 [23] and is also critical for the epithelial-injury model [15] and radiation-induced lung fibrosis [24], so is CCL2/CCR2 the magic target? It may not be that simple. Recent work in a model of interleukin-17-dependent herpesvirus-induced fibrosis post-stem cell transplant surprisingly showed that CCR2 loss made disease worse [25], suggesting significant differences in fibrosis related to pathogen stimulus versus sterile injury. Furthermore, a phase 2 trial of an anti-CCL2 therapy was stopped early for poor outcomes in idiopathic pulmonary fibrosis [26] and unexpectedly resulted in higher levels of CCL2 in patients. Targeting the receptor is likely to be far more efficacious given that CCR2 can bind multiple ligands, yet no reports of CCR2-directed therapy in lung fibrosis exist. However, there is a trial of a CCR2/ CCR5 dual antagonist being conducted in liver fibrosis [27, 28]. 
Identification of IMs as a major driver of radiation-induced pulmonary fibrosis [16] provides new CSF1R-targeted therapeutic strategies. CSF1R is also highly expressed in tumour-associated macrophages which are thought to be tumour-permissive and immunosuppressive [29]. Clinical trials of CSF1R inhibitors in cancer therapy are underway [30] and one could imagine this therapy repurposed for radiation-induced lung fibrosis. It will be interesting to see if future studies in bleomycin and epithelial cell injury models show benefit following CSF1R neutralisation to help further clarify questions of origin versus location. While the Meziani et al. [16] study specifies the importance of M2-like IMs in radiation-induced pulmonary fibrosis, IMs are strongly immunosuppressive, and a total elimination causes hypersensitivity to asthma induction [31] and accelerates acute graft versus host disease in haematopoietic stem cell transplant settings [32]. Thus, caution is warranted when considering IM depletion strategies as therapeutics. While the jury is "in" regarding the role of macrophages in lung fibrosis, we still need more research to understand how, when and where to target these cells for therapeutic benefit.

\section{References}

1 Carver JR, Shapiro CL, Ng A, et al. American Society of Clinical Oncology clinical evidence review on the ongoing care of adult cancer survivors: cardiac and pulmonary late effects. J Clin Oncol 2007; 25: 3991-4008.

2 Raghu G, Collard HR, Egan JJ, et al. An official ATS/ERS/JRS/ALAT statement: idiopathic pulmonary fibrosis: evidence-based guidelines for diagnosis and management. Am J Respir Crit Care Med 2011; 183: 788-824.

3 Raghu G, Anstrom KJ, King TE Jr, et al. Prednisone, azathioprine, and N-acetylcysteine for pulmonary fibrosis. N Engl J Med 2012: 366: 1968-1977.

4 Giridhar P, Mallick S, Rath GK, et al. Radiation induced lung injury: prediction, assessment and management. Asian Pac J Canc Prevent 2015; 16: 2613-2617.

5 Byrne AJ, Maher TM, Lloyd CM. Pulmonary macrophages: a new therapeutic pathway in fibrosing lung disease? Trends Mol Med 2016; 22: 303-316.

6 Misharin AV, Morales-Nebreda L, Mutlu GM, et al. Flow cytometric analysis of macrophages and dendritic cell subsets in the mouse lung. Am J Respir Cell Mol Biol 2013; 49: 503-510.

7 Zaynagetdinov R, Sherrill TP, Kendall PL, et al. Identification of myeloid cell subsets in murine lungs using flow cytometry. Am J Respir Cell Mol Biol 2013; 49: 180-189.

8 Gibbings SL, Thomas SM, Atif SM, et al. Three unique interstitial macrophages in the murine lung at steady state. Am J Respir Cell Mol Biol 2017; 57: 66-76.

9 Guilliams M, De Kleer I, Henri S, et al. Alveolar macrophages develop from fetal monocytes that differentiate into long-lived cells in the first week of life via GM-CSF. J Exp Med 2013; 210: 1977-1992.

10 Schneider C, Nobs SP, Kurrer M, et al. Induction of the nuclear receptor PPAR-gamma by the cytokine GM-CSF is critical for the differentiation of fetal monocytes into alveolar macrophages. Nat Immunol 2014; 15: 1026-1037.

11 Misharin AV, Morales-Nebreda L, Reyfman PA, et al. Monocyte-derived alveolar macrophages drive lung fibrosis and persist in the lung over the life span. J Exp Med 2017; 214: 2387-2404.

12 Mould KJ, Barthel L, Mohning MP, et al. Cell origin dictates programming of resident versus recruited macrophages during acute lung injury. Am J Respir Cell Mol Biol 2017; 57: 294-306.

13 Schulz C, Gomez Perdiguero E, Chorro L, et al. A lineage of myeloid cells independent of Myb and hematopoietic stem cells. Science 2012; 336: 86-90.

14 Bain CC, Bravo-Blas A, Scott CL, et al. Constant replenishment from circulating monocytes maintains the macrophage pool in the intestine of adult mice. Nat Immunol 2014; 15: 929-937.

15 Osterholzer JJ, Olszewski MA, Murdock BJ, et al. Implicating exudate macrophages and Ly-6C(high) monocytes in CCR2-dependent lung fibrosis following gene-targeted alveolar injury. J Immunol 2013; 190: 3447-3457.

16 Meziani L, Mondini M, Petit B, et al. CSF1R inhibition prevents radiation pulmonary fibrosis by depletion of interstitial macrophages. Eur Respir J 2018; 51: 1702120.

17 Martinez FO, Gordon S. The M1 and M2 paradigm of macrophage activation: time for reassessment. F1000Prime Rep 2014; 6: 13.

18 Stein $\mathrm{M}$, Keshav $\mathrm{S}$, Harris $\mathrm{N}$, et al. Interleukin 4 potently enhances murine macrophage mannose receptor activity: a marker of alternative immunologic macrophage activation. J Exp Med 1992; 176: 287-292.

19 Byrne AJ, Mathie SA, Gregory LG, et al. Pulmonary macrophages: key players in the innate defence of the airways. Thorax 2015; 70: 1189-1196.

20 Braga TT, Agudelo JS, Camara NO. Macrophages during the fibrotic process: M2 as friend and foe. Front Immunol 2015; 6: 602.

21 Murray PJ, Allen JE, Biswas SK, et al. Macrophage activation and polarization: nomenclature and experimental guidelines. Immunity 2014; 41: 14-20.

22 Phan SH, Armstrong G, Sulavik MC, et al. A comparative study of pulmonary fibrosis induced by bleomycin and an $\mathrm{O}_{2}$ metabolite producing enzyme system. Chest 1983: 83: Suppl. 5, 44S-45S.

23 Moore BB, Paine R III, Christensen PJ, et al. Protection from pulmonary fibrosis in the absence of CCR2 signaling. J Immunol 2001: 167: 4368-4377.

24 Groves AM, Johnston CJ, Williams JP, et al. Role of infiltrating monocytes in the development of radiation-induced pulmonary fibrosis. Radiat Res 2018; in press [https://doi.org/10.1667/RR14874.1].

25 Gurczynski SJ, Procario MC, O’Dwyer DN, et al. Loss of CCR2 signaling alters leukocyte recruitment and exacerbates gamma-herpesvirus-induced pneumonitis and fibrosis following bone marrow transplantation. Am J Physiol Lung Cell Mol Physiol 2016; 311: L611-L627.

26 Raghu G, Martinez FJ, Brown KK, et al. CC-chemokine ligand 2 inhibition in idiopathic pulmonary fibrosis: a phase 2 trial of carlumab. Eur Respir J 2015; 46: 1740-1750.

27 Friedman S, Sanyal A, Goodman Z, et al. Efficacy and safety study of cenicriviroc for the treatment of non-alcoholic steatohepatitis in adult subjects with liver fibrosis: CENTAUR Phase $2 \mathrm{~b}$ study design. Contemporary Clinical Trials 2016; 47: 356-365. 
28 Lefebvre E, Gottwald M, Lasseter K, et al. Pharmacokinetics, safety, and CCR2/CCR5 antagonist activity of cenicriviroc in participants with mild or moderate hepatic impairment. Clin Transl Sci 2016; 9: 139-148.

29 Laoui D, Van Overmeire E, De Baetselier P, et al. Functional relationship between tumor-associated macrophages and macrophage colony-stimulating factor as contributors to cancer progression. Front Immunol 2014; 5: 489.

30 Cannarile MA, Weisser M, Jacob W, et al. Colony-stimulating factor 1 receptor (CSF1R) inhibitors in cancer therapy. J Immunother Cancer 2017; 5: 53.

31 Bedoret $\mathrm{D}$, Wallemacq $\mathrm{H}$, Marichal $\mathrm{T}$, et al. Lung interstitial macrophages alter dendritic cell functions to prevent airway allergy in mice. J Clin Invest 2009; 119: 3723-3738.

32 MacDonald KP, Palmer JS, Cronau S, et al. An antibody against the colony-stimulating factor 1 receptor depletes the resident subset of monocytes and tissue- and tumor-associated macrophages but does not inhibit inflammation. Blood 2010; 116: 3955-3963. 